normal control subjects. (mRNA level: DCM :0.37 \pm 0.08 , NC: $0.19 \pm 0.03, \mathrm{p}<0.01$; protein level: DCM: $208.94 \pm 50.31 \mathrm{pg} / \mathrm{ml}$; NC: $175.69 \pm 44.56 \mathrm{pg} / \mathrm{ml} ; \mathrm{p}<0.01$ ) (2) The IL-35 subuint-EBI3 or its protein level was significantly decreased in DCM patients compared with normal control subjects. (EBI3 mRNA level: DCM: $0.15 \pm 0.03$, NC: $0.33 \pm 0.07, \mathrm{p}<0.01$; protein level: DCM: $128.68 \pm 24.08 \mathrm{pg} / \mathrm{ml}$, NC: $179.73 \pm 43.89 \mathrm{pg} / \mathrm{ml}, \mathrm{p}<0.01$ ) (3) The secretion of IL-34 was markedly correlation with the secretion of IL-35 ( $\mathrm{r}=-0.490, \mathrm{p}$ $<0.01$ ). (4) The protein level of IL-34 in DCM patients had a positive correlation with heart function $(\mathrm{r}=0.598, \mathrm{P}<0.01)$. (5) The protein level of IL-35 in DCM patients had a negative correlation with heart function $(r=-0.839, p<0.01)$

Conclusion The ability to express IL-34 and IL-35 protein or mRNA in PBMCs is abnormal and the change strongly correlates with ejection fraction and heart function of DCM patients.

\section{e0103 THE HINDIII POLYMORPHISM IN THE LIPOPROTEIN LIPASE GENE PREDICTS TYPE 2 DIABETES RISK AMONG CHINESE ADULTS}

doi:10.1136/hrt.2010.208967.103

${ }^{1}$ Oi Yue, ${ }^{2}$ Liu Jing, ${ }^{2}$ Wang Wei, ${ }^{2}$ Wang Miao, ${ }^{2}$ Sun Jiayi, ${ }^{2}$ Liu Jun, ${ }^{2}$ Li Yan, ${ }^{2}$ Qin Lanping, ${ }^{2}$ Wu Zhaosu, ${ }^{2}$ Zhao Dong. ${ }^{1}$ Capital Medical University Beijing Anzhen Hospital Beijing Institute of Heart Lung And Blood Vessel Diseases Beijing China; ${ }^{2}$ Capital Medical University Beijing Anzhen Hospital Beijing Institute of Heart Lung And Blood Vessel Diseases Beijing China

Objective To explore whether the HindIII polymorphism in the lipoprotein lipase (LPL) gene has a potential role in susceptibility to type 2 diabetes, and whether this relation is influenced by regulating LPL or other risk factors.

Research design and methods Overall, 654 Han Chinese adults were recruited from a community-based cross-sectional study. Genotyping was performed using the PCR-RFLP technique. Pre-heparin LPL (PrLPL) and other metabolic variables were measured using standard methods.

Results Individuals with the HindIII $\mathrm{H}-/ \mathrm{H}-$ genotype tended to have higher PrLPL and lower triglyceride (TG) levels but an unexpected higher prevalence of type 2 diabetes compared with carriers with the $\mathrm{H}+\mathrm{H}+$ genotype. The association between the $\mathrm{H}-/ \mathrm{H}-$ genotype and diabetes risk remained unchanged across all subgroups of diabetesrelated risk factors and PrLPL. In an additive model, the $\mathrm{H}-/$ $\mathrm{H}-$ genotype conferred 178\% increased risk [OR:2.78; 95\% CI 1.04 to 7.47] for diabetes after controlling for age and sex. The strength of this association increased further after adjusting for other traditional risk factors, and for PrLPL (OR=4.06; 95\% CI=1.35 to 12.23). Furthermore, the $\mathrm{H}-/ \mathrm{H}-$ genotype was also associated with an increased risk of dysglycemia defined as insulin resistance plus diabetes.

Conclusions This study revealed that Chinese adults with the LPL gene HindIII $\mathrm{H}-/ \mathrm{H}-$ genotype had a significantly increased risk of type 2 diabetes compared with individuals with other genotypes, even if they had favourable lipid profiles.

\section{E0104 MATCHED CASE-CONTROL STUDY ON MECHANISM OF RADIAL ARTERY SPASM}

doi:10.1136/hrt.2010.208967.104

Jia Dean, Zhou Yujie, Shi Dongmei, Wang Zhijian, Yang Shiwei, Liu Xiaoli. Beijing Anzhen Hospital Capital Medical University

Objects Radial artery (RAS) spasm is the most common complication in transradial coronary angiography and intervention. The aim of this study is to preliminary discuss the relationship between vaso-active substances and RAS, find out the mechanism of RAS, and provide theoretic evidence for the solution of RAS prevention.
Methods This is a prospective, matched case-control study. The patients who received transradial coronary angiography were enrolled. The patients who suffered from RAS during the procedure were enrolled, and the patients without RAS were matched 1:2 according to same gender, similar age within 2 years. The diagnostic criteria are clinical definition of RAS based on a questionnaire which was documented by angiography. Blood samples were obtained before the procedure, and were tested for nitric oxide, endothelin-1, prostacyclin, thromboxane A2 and norepinephrine using enzymelinked-immunosorbent assay. The concentration of each vaso-active substance was compared and multi logistic regression was made to find out the risk factors of RAS.

Results 30 patients suffered form RAS and 60 patients without RAS were enrolled. of all the clinical and procedural characteristics, successful access at first attempt $(46.7 \%$ vs $75.0 \%, p=0.010)$ and ratio of severe pain at cannulation ( $13.3 \%$ vs $1.7 \%, p=0.041)$ were different between the RAS group and the control group, the others were of no difference. The concentration of nitric oxide $(64.5512 \pm 24.2963 \mathrm{vs}$ $57.6385 \pm 20.1472, p=0.426)$ and thromboxane A2 $(0.9040 \pm 0.2158$ vs $0.7364 \pm 0.2256, p=0.372$ ) was of no difference between the RAS group and the control group. The concentration of endothelin-1 $(276.3739 \pm 85.1481$ vs $78.5275 \pm 23.6323, p<0.001)$ and norepinephrine (193.7551 \pm 41.8509 vs $54.4108 \pm 17.8031, p=0.006)$ was higher, prostacyclin $(8.1947 \pm 3.2692$ vs $14.5436 \pm 5.5867, p=0.041)$ was lower in RAS group. Multiple regression showed that endothelin-1 (OR 2.714, 95\% CI 1.329 to 4.984, $\mathrm{p}=0.005$ ) and norepinephrine (OR 4.285, 95\% CI 2.219 to $10.372, p=0.014$ ) were the risk factors of RAS during the procedure.

Conclusions Among the vaso-active substances, the concentration of nitric oxide and thromboxane A2 was of no difference, prostacyclin was lower and endothelin-1, norepinephrine was higher in RAS patients than in patients without RAS. Multiple regression showed that endothelin-1 and norepinephrine were the risk factors of RAS during the procedure.

\section{E0105 IN VITRO BLOCKADE OF OESTROGEN RECEPTOR PROMOTES THE PROLIFERATION OF VASCULAR SMOOTH MUSCLE CELLS}

doi:10.1136/hrt.2010.208967.105

${ }^{1}$ Hong He, ${ }^{2}$ Fa-Lin Yang, ${ }^{3}$ Xin Wang, ${ }^{4}$ Hui Zhu, ${ }^{4}$ Jinghuan Huang, ${ }^{4}$ Zimo Liu. ${ }^{1}$ Department of Cardiology, Qilu Department of Cardiology, Qilu Hospital, University of Shandong, Jinan, China; ${ }^{2}$ Clinical Laboratory, Qilu Hospital, University of Shandong, Jinan, China; ${ }^{3}$ Department of Clinical Medicine, the Second Affiliated Hospital, University of Shandong, Jinan, China; ${ }^{4}$ Department of Cardiology, Qilu Hospital, University of Shandong, Jinan, China

Background The proliferation of vascular smooth muscle cells (VSMCs) is a key event in the development of atherosclerosis. Oestrogen receptor is expressed in VSMCs. In vivo studies have shown that reduced levels of oestrogen receptor associate with atherosclerosis in females. Accordingly, we performed a series of experiments to test the hypothesis that blocking oestrogen receptor could enhance the proliferation of VSMCs in vitro.

Methods and results ICI182, 780, a pure oestrogen receptor antagonist, has been shown to block oestrogen receptor completely. When VSMCs isolated from rat aorta were cultured in the presence of ICI182, 780 , the cellular growth augmented significantly in a dose-dependent manner. An increase in proliferating cell nuclear antigen (PCNA)positive cells was also observed in VSMCs treated with ICI182, 780. Flow cytometry demonstrated that the S-phase progression of cell cycle in the VSMCs was promoted significantly by ICI182, 780, this effect was associated with an increase in cyclin D1 expression.

Conclusions These findings demonstrate that in vitro blockade of oestrogen receptor promotes the growth of VSMCs, suggesting that oestrogen receptor expressed in arteries acts to inhibit the 
proliferation of VSMCs and play a functional role in atheroprotection. Efforts aiming at enhancing oestrogen receptor expression and/or activity may prove to be an attractive alternative therapy against atherosclerosis.

\section{e0106 THE EFFECT OF ADENOSINE AND ISCHAEMIA POSTCONDITIONING ON MMP-2 AND MMP-9 IN RABBIT ISCHAEMIA REPERFUSION MYOCARDIAL}

doi:10.1136/hrt.2010.208967.106

${ }^{1}$ HeXiaonan, ${ }^{2} Y u$ Chen, ${ }^{3}$ Li Shumei. ${ }^{1}$ Department of Cardiology, TianJin Chest Hospital, TianJin; ' ${ }^{2}$ epartment of Cardiology, China-Japan Union Hospital, Jilin University, Changchun; ${ }^{3}$ Department of Cardiology, the Second Hospital of Jili University, Changchun

Objective To observe the effect of adenosine and ischaemia postconditioning on MMP-2 and MMP-9 in rabbit ischaemia reperfusion myocardial.

Methods The rabbits were divided into four groups in basic experiment: control group, antagon group, postconditiong group and adenosine group. The activity of MMP-2 and MMP-9 was observed by gelatin zymography and the expression of MMP-2 and MMP-9 was observed by RT-PCR and Western Blot.

Results The results of RT-PCR showed that the light density of MMP-2/GAPDH $(0.76 \pm 0.22)$ in adenosine group and postconditioning group was slightly lower than that of control group and antagon group $0.80 \pm 0.20$ ( $p>0.05)$. The light density of MMP$2 / \mathrm{GAPDH}(0.77 \pm 0.12)$ in adenosine group and postconditioning group was significanty lower than that of control group and antagon group $1.30 \pm 0.10(p<0.01)$. There was no dramitic difference between adenosine group and postconditioning group. The results of Western blot showed that MMP-9 and MMP-2 in adenosine group and postconditioning group was lower than control group and antagon group. The results of Zymography revealed that the light density of MMP-9 in adenosine group and postconditioning group much lower than those in control group and antagon group $(p<0.05)$. There was no difference between adenosine group and postconditioning ( $p>0.05)$.

Conclusions Adenosine and postconditioning can decrease the expression and the activity of MMP-9 and inhibit the inflammation, relieving the ischaemia reperfusion injury.

\section{e0107 CARDIAL PROTECTIVE EFFECTS OF DIFFERENT DOSAGE ATORVASTATIN IN PATIENTS WITH STABLE ANGINA AFTER PERCUTANEOUS CORONARY INTERVENTION}

doi:10.1136/hrt.2010.208967.107

${ }^{1}$ Jin-Zi Su, ${ }^{2}$ Guang-Ling Chen, ${ }^{1}$ Wen-Qin Cai. 'Department of Cardiology, First Affiliated Hospital, Fujian Medical University, Fuzhou, ${ }^{2}$ Ming Dong Hospital, Fujian

Background The incidence of myocardial injury limits the clinical outcomes of percutaneous coronary intervention (PCI). This randomised controlled study was designed to evaluate the protective effects of pretreatment atorvastatin on myocardial injury and inflammatory reaction after PCI.

Methods 82 patients with chronic stable angina without previous statins treatment in 2 months before PCI were randomised to receive atorvastatin $10 \mathrm{mg} / \mathrm{qn}$ (group $\mathrm{A}, \mathrm{n}=27$ ), $20 \mathrm{mg} / \mathrm{qn}$ (group $\mathrm{B}$, $\mathrm{n}=28$ ) or $40 \mathrm{mg} / \mathrm{qn}$ (group $\mathrm{C}, \mathrm{n}=27$ ) treatment for 3 days before PCI. CK-MB, cTnI, hsCRP, IL-6, sICAM-1 were measured at baseline, 8 and $24 \mathrm{~h}$ after the procedure. 1-month clinical follow-up was obtained by office visit in all patients.

Results The peak levels of CK-MB and cTnI were increased significantly in all three groups $24 \mathrm{~h}$ after PCI (all $\mathrm{p}<0.05)$. Either elevation above the upper normal limit (UNL) or $>3 \times$ UNL of $c T n I$, there were significant differences between group $A$ and $B(p<0.05)$, and between group $A$ and $C(p<0.05)$, but no difference between group $B$ and $C$ $(p>0.05)$. Similarly changes were also found in CK-MB. The level of IL-6, sICAM-1 and hsCRP $8 \mathrm{~h}$ after PCI were higher than those before PCI (all $p<0.05)$. There were significant differences in IL- 6 and hsCRP among the three groups (all $\mathrm{p}<0.05$ ), but no significant difference in sICAM-1 ( $p>0.05)$. The level of hsCRP and sICAM-1 $24 \mathrm{~h}$ after PCI were higher than those $8 \mathrm{~h}$ after PCI in all three groups (all $p<0.05)$, but IL- 6 significantly decreased $(p<0.05)$. There were significant differences among the three groups (all $\mathrm{p}<0.05$ ). No serious cardiovascular events occurred during follow-up.

Conclusion Even short term pretreatment with atorvastatin before PCI may reduce procedural myocardial injury by reducing inflammatory factors in chronic stable angina patients.

\section{Q0108 ESTROGEN INDUCES RECOVERY OF INJURED ARTERY ENDOTHELIUM BY MOBILISING ENDOTHELIAL PROGENITOR CELL}

doi:10.1136/hrt.2010.208967.108

Xiaohui Zhao, Yangguang Yin, Yuqiang Fang, Jianfei Chen, Bin cui, Lan Huang. Cardiovascular Disease Research Center, Xinqiao Hospital, Third Military Medical University, Chongqing, China

Introduction Mobilization of endothelial progenitor cells (EPC) restores endothelial function, representing a novel therapeutic direction for injured blood vessel recovery. The present study was designed to determine the effect of oestrogen on EPC mobilisation and regeneration of endothelium in mice.

Methods Variectomy is performed before treatment of $17 \beta$-oestradiol, 17 $\beta$-oestradiol combination with oestrogen receptor agonistICI182780 and 17 $\beta$-oestradiol with PI3K blockers- LY294002. Then, carotid artery injury was preformed and neointima was evaluated by H.E staining. 1 and 3 days later, mobilisation of EPCs was evaluated by FACS as double positive of Sca-1/VEGFR-2. Evens blue was injected and area of reendothelization was calculated after 7 days. To trace EPCs in vivo, $1 \times 10^{6}$ autologous spleen-derived EPCs were labelled with DAPI and transplanted through tail vein.

Results 1 or 3 days after carotid artery injury, EPCs of peripheral blood were $0.42 \pm 0.135 \% \quad(n=6), 1.47 \pm 0.38 \%(n=5) \quad$ (ovariectomy $+E 2)$ $0.13 \pm 0.024 \%(n=6), 0.25 \pm 0.024 \%(n=6)$ (ovariectomy); $0.43 \pm 0.16 \%$ $(\mathrm{n}=6), 0.65 \pm 0.21 \%(\mathrm{n}=4)$ (non-ovariectomy); $0.12 \pm 0.019 \% \quad(\mathrm{n}=6)$, $0.25 \pm 0.062 \%(n=6)$ (ovariectomy $+E 2+L Y)$ and $0.12 \pm 0.019 \%(n=6)$, $0.24 \pm 0.067 \%(\mathrm{n}=6)$ (ovariectomy $+\mathrm{E} 2+\mathrm{ICI})$. Area of re-endothelization were (ovariectomy, $28.33 \pm 13.49 \%, \mathrm{n}=5$ ) vs (ovariectomy $+\mathrm{E} 2$, $69.53 \pm 14.14 \%, \mathrm{n}=5$ ) vs (non-ovariectomy, $83.11 \pm 7.94 \%, \mathrm{n}=4$ ) $(\mathrm{p}<0.01)$. In vivo tracing experiment detected blue fluorescence cells in injured sites that were also positive of CD31, indicating EPCs homing to target sites.

Conclusion Ooestrogen can induce EPCs mobilisation through ERs/ PI3K pathway which is helpful to promote endothelium recovery of injured carotid artery.

\section{e0109 THE EFFECTS OF C-REACTIVE PROTEIN ON TOLL-LIKE RECEPTOR 4 SIGNAL TRANSDUCTION ON CD14+ MONOCYTE}

doi:10.1136/hrt.2010.208967.109

Jinlai Liu, Long Peng, Yanting Luo. Department of Cardiology, The Third Affiliated Hospital, Sun Yat-sen University, Guangzhou, China

Objective To observe the effects of C-reactive protein (CRP) on Tolllike receptor 4 (TLR4) expression in $\mathrm{CD} 14^{+}$monocyte in human, and the role of CRP in the inflammatory mechanism.

Methods $\mathrm{CD}_{14}{ }^{+}$monocytes were isolated from blood in healthy volunteers by the Ficoll density gradient and stimulated by CRP 